\title{
Experimental Fusion of Contrast Enhanced High-Field Magnetic Resonance Imaging and High-Resolution Micro-Computed Tomography in Imaging the Mouse Inner Ear
}

\author{
S. Allen Counter ${ }^{1, *}$, Peter Damberg ${ }^{2}$, Sahar Nikkhou Aski ${ }^{2}$, Kálmán Nagy ${ }^{2}$, Cecilia Engmér \\ Berglin $^{3}$, and Göran Laurell ${ }^{4}$
}

\begin{abstract}
${ }^{I}$ Neurology Department, Harvard University Biological Laboratories, Cambridge, MA 02138, USA; ${ }^{2}$ Karolinska Experimental Research Imaging Center, Karolinska Universitetssjukhuset Solna, Sweden; ${ }^{3}$ Department of CLINTEC Karolinska Institutet, 17176 Stockholm, Sweden; ${ }^{4}$ Department of Surgical Sciences Uppsala University, Uppsala, Sweden
\end{abstract}

\begin{abstract}
Objective: Imaging cochlear, vestibular, and 8th cranial nerve abnormalities remains a challenge. In this study, the membranous and osseous labyrinths of the wild type mouse inner ear were examined using volumetric data from ultra high-field magnetic resonance imaging (MRI) with gadolinium contrast at 9.4 Tesla and high-resolution micro-computed tomography $(\mu \mathrm{CT})$ to visualize the scalae and vestibular apparatus, and to establish imaging protocols and parameters for comparative analysis of the normal and mutant mouse inner ear. Methods: For in vivo MRI acquisition, animals were placed in a Milleped coil situated in the isocenter of a horizontal 9.4 T Varian magnet. For $\mu \mathrm{CT}$ examination, cone beam scans were performed ex vivo following MRI using the $\mu \mathrm{CT}$ component of a nanoScan PET/CT in vivo scanner. Results: The fusion of Gd enhanced high field MRI and high-resolution $\mu \mathrm{CT}$ scans revealed the dynamic membranous labyrinth of the perilymphatic fluid filled scala tympani and scala vestibule of the cochlea, and semicircular canals of the vestibular apparatus, within the $\mu \mathrm{CT}$ visualized contours of the contiguous osseous labyrinth. The ex vivo $\mu \mathrm{CT}$ segmentation revealed the surface contours and structural morphology of each cochlea turn and the semicircular canals in 3 planes. Conclusions: The fusion of ultra high-field MRI and high-resolution $\mu \mathrm{CT}$ imaging techniques were complementary, and provided high-resolution dynamic and static visualization of the complex morphological features of the normal mouse inner ear structures, which may offer a valuable approach for the investigation of cochlear and vestibular abnormalities that are associated with birth defects related to genetic inner ear disorders in humans.
\end{abstract}

Keywords: Cochlea, Vestibular, Magnetic Resonance Imaging, Micro Computed Tomography, Gadolinium.

\section{INTRODUCTION}

Magnetic resonance imaging (MRI) of the inner ear serves as an excellent clinical and research tool for investigation of pathophysiology of the cochlear, vestibular apparatus, 8th cranial nerve, and the pharmacokinetics of drug delivery in the perilymphatic and endolymphatic spaces $[1,2]$. Conventional MRI at 1.5 Tesla is used clinically to examine the cochlea, internal auditory canal and cerebellopontine angle in patients with suspected vestibular schwannoma, endolymphatic hydrops, and some facial nerve disorders [3-5]. Clinical MRI of the perilymphatic compartments of the inner ear has been enhanced through intravenous, trans-tympanic, and round window injection of the paramagnetic contrast agent gadolinium (Gd) for enhanced visualization of the status of the cochlea, semicircular canals and retro-cochlear structures $[1,4,5]$.

Computed tomography (CT) has also been used clinically for examination of the osseous labyrinth of the inner ear in investigations of genetically based vestibulo-cochlear dis-

*Address correspondence to this author at the Department of Neurology, Harvard University, Biological Laboratories, Cambridge, MA 02138, USA; E-mail: allen_counter@harvard.edu orders such as inner ear dehiscence, lateral semicircular canal malformation, and X-linked mixed deafness with perilymph gusher, as well as dysmorphogenesis and disabling disequilibrium [6,7]. Combined MRI and CT fusion techniques have been used to examine disorders of the inner ear and tumors in the cerebellopontine angle, and have been found to be complementary techniques $[2,8,9]$.

In comparative animal studies, Gd enhanced high-field experimental MRI has been used in in vivo and in vitro investigations of the cochlea and vestibular apparatus of the mammalian inner ear [10-12]. The effective ex-perimental use of the mouse model in Gd enhanced high-field MRI studies of the inner ear has been demonstrated in recent studies $[12,13]$.

In recent experimental investigations of the inner ear of murine species, micro-computed tomography $(\mu \mathrm{CT})$ has been used effectively as a research tool for in vivo and ex vivo investigations of anatomical structure and genetically associated malformations of the membranous and osseous labyrinths $[14,15]$. Combined MRI and $\mu \mathrm{CT}$ experimental techniques have been used to create stereotaxic atlases of the normal mouse skull and brain, to examine patterns in skull morphology, brain shape and inner ear volume in Crouzon 
syndrome mice after fixation in 4\% paraformaldehyde [1619], and to investigate the mouse spinal cord in vivo [20].

In a recent investigation of the dynamic morphology of the wild-type mouse inner ear using ultra high-field (9.4 Tesla) MRI scans, the authors established the parameters associated with the temporal and spatial uptake of $\mathrm{Gd}$, and performed three-dimensional reconstruction of the scala tympani and scala vestibule of the cochlea, and the anatomical orientation of the vestibular apparatus [13]. The study demonstrated that high-field experimental MRI could be used in vivo to monitor the temporal and spatial uptake of intravenously injected $\mathrm{Gd}$, and to visualize the entire perilymphatic space, but not the $\mathrm{Gd}$ impermeable endolymphatic labyrinth of the wild type mouse cochlea and semicircular canals [13].

The objective of the present study was to conduct a combined ultra high field MRI investigation of the complex morphological features of the $\mathrm{Gd}$ filled perilymphatic labyrinth in conjunction with a matched $\mu \mathrm{CT}$ examination of the contiguous osseous labyrinth of the cochlea and vestibular apparatus of the normal mouse inner ear. The overarching purpose was to establish normal protocols and parameters for experimental $\mathrm{MRI}$ and $\mu \mathrm{CT}$ fusion in investigations of inner ear disorders in mice with genetic disorders analogous to genetic conditions found in humans, and to examine the symmetry between the $\mu \mathrm{CT}$ and MR images.

\section{METHODS AND MATERIALS}

All animal experiments were approved by the Ethical Committee for Animal Research in Stockholm, Sweden 2014. Ten normal male mice (wild-type BALB-c), 13 weeks old, weighing 22-25 grams, were used in this experimental investigation. Anesthesia was induced in an animal chamber with 5\% Isoflurane and 1:1 mixture of oxygen and air. Animals were then positioned prone in a mouse swift system (Varian Inc.) and anesthesia was maintained during the scanning using 1-2\% Isoflurane in 1:1 mixture of oxygen and air, with a total flow of $1 \mathrm{~L} / \mathrm{min}$. The gadolinium contrast agent Gadovist [Gadobutrol C18H31GdN4O9; Bayer Schering Pharma] was administered intravenously (IV) using the tail vein in a single dose of $3 \mathrm{mmol} / \mathrm{kg}$ using an infusion pump (Harvard apparatus, Standard infuse/Withdraw PHD 2000, Massachusetts). For high field MR image acquisition, the anesthetized animals were placed in a Milleped coil (Varian Inc.) with an inner diameter of $30 \mathrm{~mm}$, and situated in the isocenter of a horizontal 9.4 $\mathrm{T}$ Varian magnet equipped with a $12 \mathrm{~cm}$ inner diameter gradient system with maximum gradient strength of $600 \mathrm{mT} / \mathrm{m}$. T1- weighted 2D images were acquired using a Fast Spin Echo sequence. Acquisition Parameters: TR: $500 \mathrm{~ms}$; effective TE: $10 \mathrm{~ms}$; echo train length (ETL): 4; echo spacing: $92.4 \mathrm{~ms}$; number of averages: 32 ; data matrix: $256 \times 256$; field of view (FOV): $19.9 \times 20 \mathrm{~mm}^{2}$; resolution $\left(0.078 \times 0.078 \mathrm{~mm}^{2}\right)$; with the slice-select gradient being applied from left to right. T1weighted 3D images were acquired with a Gradient Echo 3D sequence. TR: $7.95 \mathrm{~ms}$; TE: $1.97 \mathrm{~ms}$; number of averages: 1; data matrix: $192 \times 384 \times 192$; FOV: $12.8 \times 30.8 \times 15.4 \mathrm{~mm} 3$; resolution: $0.067 \times 0.08 \times 0.08 \mathrm{~mm}^{3}$ (Slab thickness: 13.9 $\mathrm{mm}$, with the slice-select gradient being applied ventral to dorsal). One 3D image was acquired before injecting the contrast agent as the baseline image. Thereafter 3D images were acquired every 10 minutes after the injection using the pump with a flow rate of $100 \mu$ liters/min during uptake for 110 minutes. ImageJ version $1.43 \mu$, National Institutes of Health, 2003, and OsiriX software version 4.1.2 32-bit, a Macintosh-based medical imaging software, as well as Adobe Photoshop CS5 were used for the image processing.

For computed tomography examination, animals were euthanized with an overdose of anesthesia, and cone beam CT scans were performed ex vivo using the CT component of a nanoScan PET/CT (model CT08) (Mediso Ltd., Hungary) in-vivo scanner. Acquisition parameters were spiral trajectory with 360 projections/rotation, 4.5 fold optical magnification, $65 \mathrm{kV}$ tube voltage, $123 \mathrm{~mA}$ tube current and $600 \mathrm{~ms}$ exposure time. The data were reconstructed using a modified Feldkamp algorithm on a $20 \mu \mathrm{m}$ isotropic voxelmatrix. Manual coregistration and reorientation of the MRI and CT volumes were performed using Interview Fusion software (Mediso Ltd.). The coregistration was rigid, and after fitting an axial section of the scull, it was further optimized for one side only. No scaling was required to achieve a good match between the two volumes.

\section{RESULTS}

MRI: Gd enhanced high field MRI scans revealed the morphology of the spirals of the cochlea from base to apex, and the vestibular structures in the normal BALB-c mouse. As can be seen in Fig. (1), the contrast agent was transported through the entire perilymphatic compartments of the cochlea and the vestibular apparatus, revealing the scala tympani and scala vestibule; and the lateral, superior and anterior semicircular canals. The time course of the uptake and enhancement of the MRI followed a predictable pattern, with $\mathrm{Gd}$ saturation of the scalae in 60 minutes. The semicircular canals were visualized later than the scala tympani and scala vestibule of the cochlea, at 80 minutes. The Gd contrast agent did not penetrate the scala media.

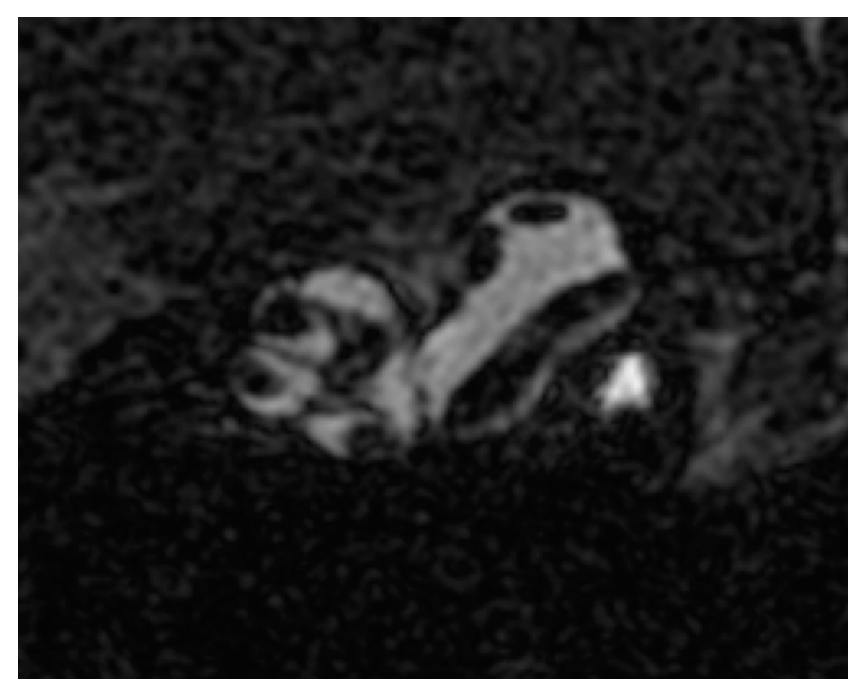

Fig. (1). 2D high resolution (9T) transverse oriented slice of the normal mouse cochlea enhanced for visualization by the IV injected Gd, showing the scala tympani and scala vestibule, and the lateral semicircular canal of the vestibular system. 


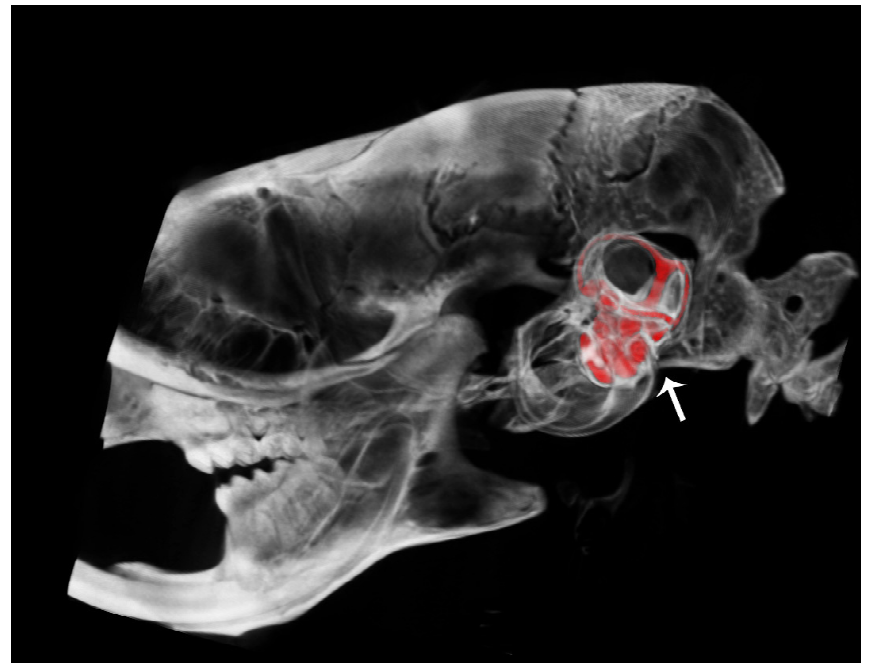

Fig. (2). Lateral view of a $\mu \mathrm{CT}$ fused with a $\mathrm{CT}$ segmentation of the perilymphatic space of the scala tympani and scala vestibuli of the cochlea and semicircular canals of the vestibular apparatus of the osseous labyrinth in the mouse inner ear.

Fig. (2) shows a lateral view of a $\mu \mathrm{CT}$ fused image with a CT segmentation of the osseous inner ear labyrinth illustrating the perilymphatic space of the scala tympani and scala vestibuli of the cochlea and the semicircular canals of the vestibular apparatus in the temporal region of the cranium (arrow) of the wild type mouse. This fused image reveals anatomical position and morphological structure of the spiral scalae of the cochlea from base to apex and the vestibular apparatus, including the lateral, anterior and posterior semicircular canals (represented in red).

Fig. (3A, B and C) illustrates $\mu \mathrm{CT}$ scans of the osseous mouse cochlea chambers (white), including (red arrows) the scala tympani in the basal turn (A), lateral view of the scala tympani, scala media, scala vestibuli and semicircular canals (B) and (C). Fig. (3) (D, E, F): $\mu$ CT fused with a CT segmentation of the perilymphatic space (red) sections of the mid-cochlea (D) of the scala tympani and scala vestibuli of the cochlea and semicircular canals of the vestibular apparatus $(\mathrm{E}, \mathrm{F})$ of the normal wild-type mouse inner ear.

Fig. (4) shows high-resolution fused MR- $\mu \mathrm{CT}$ images of the membranous and osseous labyrinths of the semicircular canals and scalae of the cochlea in the wild type mouse. This figure demonstrates an advantage of superimposed MRI$\mu \mathrm{CT}$ scans in analytical morphological observations of the mouse inner ear. Fig. (4) (A, B, C) shows marginally traceable, generally oriented MRI slices of the semicircular canals (1) and cochlea (2,3). After coregistration with the $\mu \mathrm{CT}$ and reorienting the volume to align the semicircular canals in the orthogonal viewing planes, the Gd-enhanced perilymphatic spaces became clearly visible (Fig. 4 D, E, F), revealing the lateral semicircular canal (1), and the scala vestibule and scala tympani of the cochlea $(2,3)$.

The summary composite in Fig. (5) (A, B) shows volume rendered images of the cochlear and vestibular apparatus of the wild-type mouse inner ear. Fig. (5) A illustrates the contrast enhanced perilymphatic spaces of the scala tympani (Green), scala vestibule (Green) and the scala media (red). The lateral, posterior and anterior semicircular canals (Green) are shown below the cochlea chambers in an inferolateral orientation. Fig. (5) B presents a reverse view of a $3 \mathrm{D}$ volume rendered image with emphasis on the vestibular apparatus of the mouse inner ear in the superior orientation, showing the contrast enhanced perilymphatic spaces of the lateral, posterior and anterior semicircular canals (Green) with the cochlea scalae in the inferior orientation, surrounded by the osseous labyrinth (White).
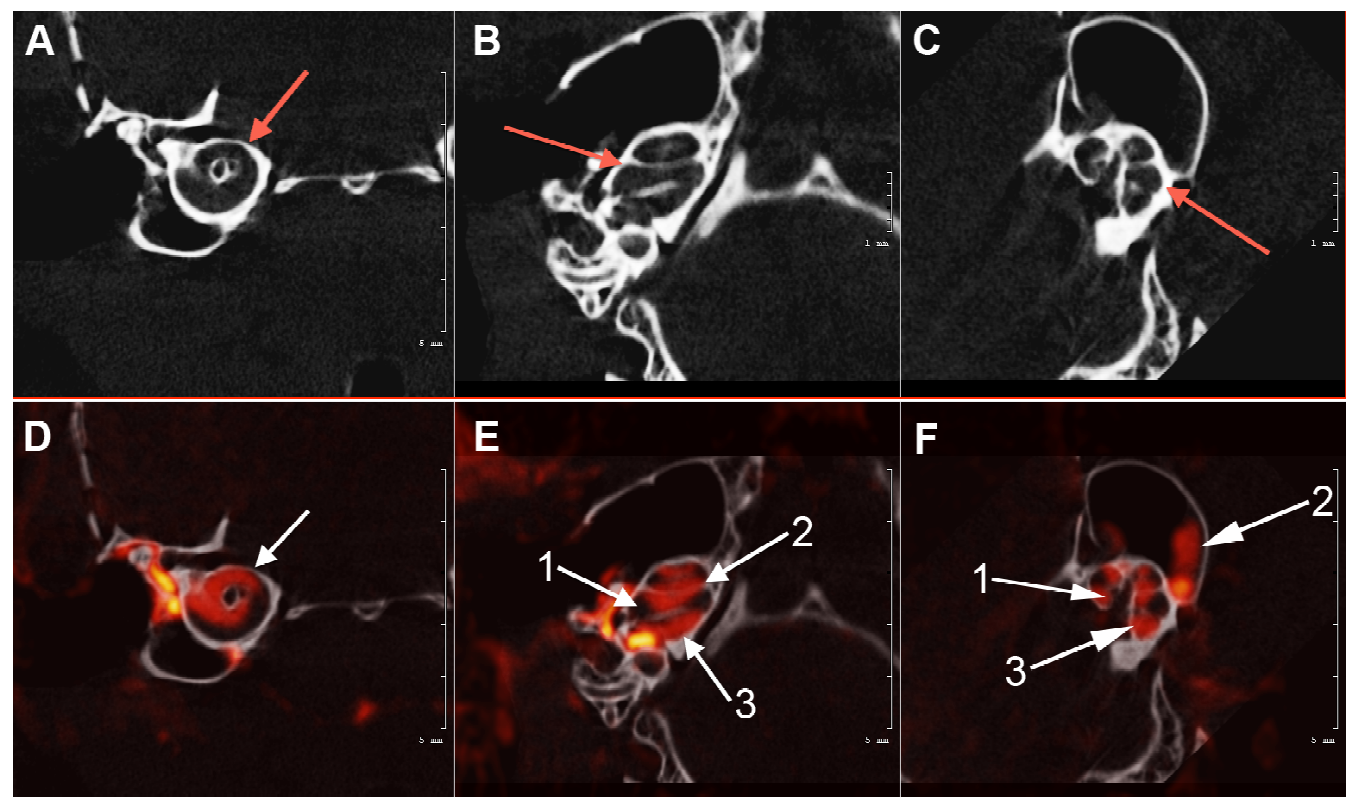

Fig. (3). Axial view of the $\mu \mathrm{CT}$ scans of the osseous mouse cochlea chambers (white) including (arrows) the scala tympani area (A), scala media, scala vestibuli, vestibular apparatus, and semicircular canals (B) and (C). Fig. (3): (D). white arrow shows $\mu$ CT fused with a CT segmentation of the perilymphatic space (red) sections of the mid-cochlea; (E). (1) Scala media; (2) scala vestibuli and Scala tympani (3) (F). Contiguous osseous labyrinth of the mouse inner ear showing the (1) scala vestibule, (2) semicircular canals of the vestibular apparatus, and (3) scala tympani of the cochlea. 


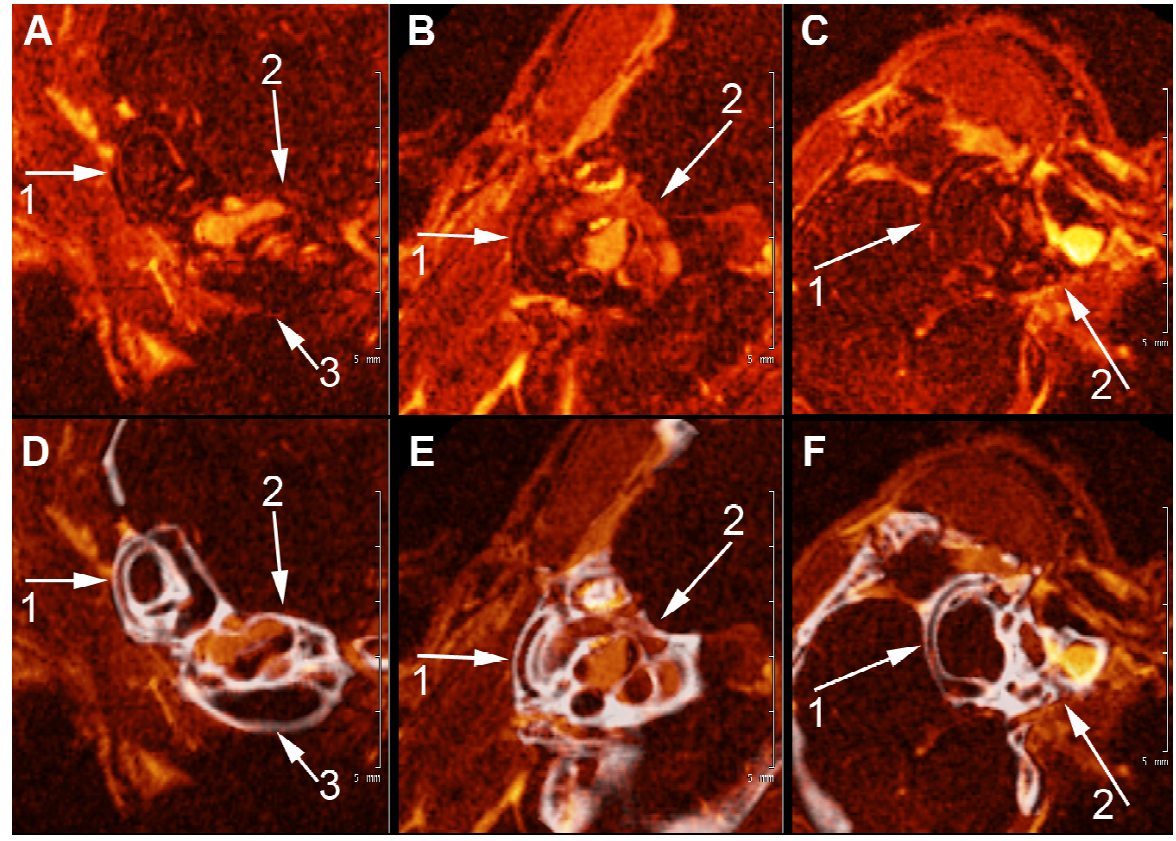

Fig. (4). A, B, and C show high field MRI Gd-enhanced images of the scala tympani, scala vestibuli and semi-circular canals of the mouse inner ear (1) semicircular canals of the vestibular apparatus, (2), scala vestibuli and scala tympani (3). 4. D, E, F show fusion images (in white) of CT segmentation of the cochlea and semicircular canals of the contiguous osseous labyrinth of the mouse inner shown in $\mathbf{A}, \mathbf{B}$, and C.

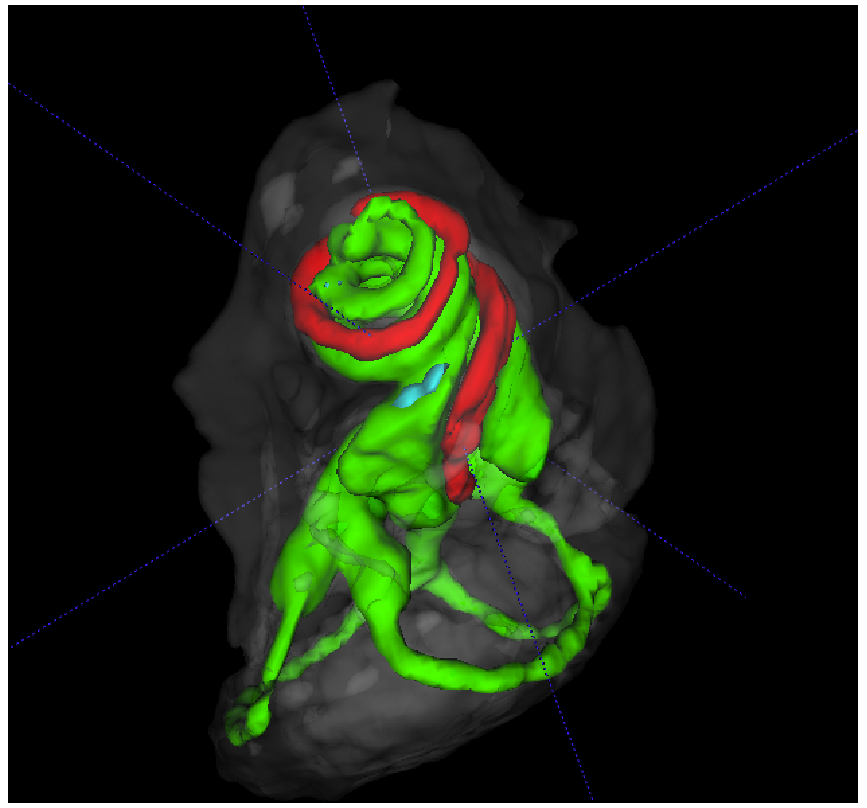

Fig. (5A). 3D volume rendered cochlear and vestibular apparatus of the mouse inner ear with focus on the cochlea, showing the contrast enhanced perilymphatic spaces of the scala tympani (Green), scala vestibule (Green) and the scala media (red), and the lateral, posterior and anterior semicircular canals (Green), surrounded by the osseous labyrinth (White). The vestibular system is seen below the cochlea.

\section{DISCUSSION}

MRI is used for clinical assessment of vertigo and sensorineural hearing loss, cerebellopontine angle tumors and hypoplasia of the cranial nerves. High field MRI has been used in animal studies to investigate experimentally

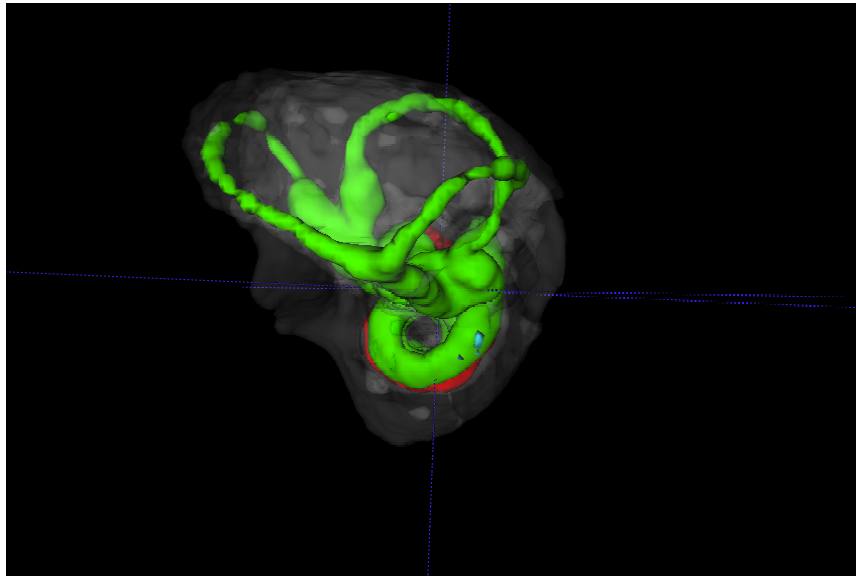

Fig. (5B). Reverse view of a $3 \mathrm{D}$ volume rendered vestibular apparatus and cochlea of the mouse inner ear with focus on the vestibular system, showing the contrast enhanced perilymphatic spaces of the lateral, posterior and anterior semicircular canals (Green), surrounded by the osseous labyrinth (White). The cochlea scalae are seen below.

induced conditions that mimic human auditory-vestibular disorders. Ultra high field MRI at 9.4 $\mathrm{T}$ has been employed recently in studies of the mouse brain and spinal cord and investigations of its potential use and safety in human subjects [21, 22]. In the present study, high-resolution MRI of the mouse inner ear was enhanced by Gd to reveal the perilymphatic spaces throughout the membranous labyrinth of the scala tympani and scala vestibule and semicircular canals. Visualization of the wild type mouse inner ear using high field strength MRI was achieved in a predictable stepwise fashion over a period of approximately 80 minutes following intravenous $\mathrm{Gd}$ injection. Gd shortens the $\mathrm{T} 1$ 
relaxation time and thereby lowers the signal to noise ratio and creates an enhanced signal/image. By the protocol used in this investigation, the initial uptake of the contrast agent was first observed in the cochlea at approximately 30 minutes post $\mathrm{Gd}$ injection. The lateral, anterior and posterior semicircular canals were visualized in succession at 60 to 80 minutes. As shown in previous investigations, the endolymphatic compartment was difficult to resolve since the Gd contrast signal is challenging to measure when there is a large gradient between adjacent compartments. It has been shown in a previous study that an intravenous injection of a radioactive tracer contrast agent may fill the endolymphatic space at a much lower concentration than what is seen in the perilymphatic compartments [23]. Improvements in high-field MRI techniques may provide better resolution of the pharmacokinetics of the comparative uptake and elimination of contrast agents between the perilymphatic and endolymphatic spaces.

Significantly, the high flip angle/short echo time used in the present study to acquire gradient echo 3D images resulted in better visualization of the inner ear morphology than the RARE technique used in previous studies. Because of the short echo time of the technique used in this study, little signal loss occurred. As a consequence, a rather linear and uniform signal increase in $\mathrm{Gd}$ within the cochlea compartments was observed. Also, the slice thickness was found to be thinner than can be achieved with the RARE sequence.

This study uniquely examined the fusion of Gd contrast enhanced ultra high field MRI combined with $\mu \mathrm{CT}$ fused with CT segmentation images of the perilymphatic membranous and osseous labyrinths, respectively of the wild type mouse inner ear. An advantage with the present study was the MRI images obtained in vivo showing that the dynamic uptake of Gd could be combined with ex vivo analysis of the bony labyrinth frame delineated by $\mu \mathrm{CT}$. Thus the combined MRI- $\mu$ CT imaging techniques were complementary, and provided high-resolution dynamic and static visualization of the morphological features of the normal mouse inner ear structures. These observations are promising in that they provide further evidence that fusion and superimposition of high field MRI and $\mu \mathrm{CT}$ will be useful in investigation and detection of cochlear or vestibular apparatus disorders in mutant mouse models that mimic birth defects in humans related to genetic inner ear disorders such as dehiscence, dilated vestibule, enlarged vestibular aqueduct, dysmorphogenesis, partial or complete semicircular canal aplasia and various malformations of the vestibulo-cochlear systems. Detailed knowledge derived from these experimental studies can be useful for development of better diagnostic tools and models for human inner ear disorders.

Tomographic images of the murine inner ear enable observations of minute aberrations in morphology of the cochlea and vestibular apparatus. The high-resolution cone beam $\mu \mathrm{CT}$ images acquired in the present study permitted unrestricted visualization of the entire normal mouse inner ear, including the osseous labyrinth, scalae of the cochlea, and the lateral, anterior and posterior semicircular canals. The use of $\mu \mathrm{CT}$ for tomographic images of the mutant mouse inner ear may enable diagnostic visualization of the vestibular apparatus in disorders such as enlarged vestibular aqueduct, dilated vestibule and other malformations in mice with genetic disorders that mimic analogous conditions found in humans with, for example Crouzon syndrome and Pendred syndrome. It is also significant that the observations in all planes showed no severe asymmetry between the $\mu \mathrm{CT}$ and the high field MRI images of the cochlea and vestibular apparatus.

The demonstrated experimental fusion of imaging techniques used in this study offers useful methodology for detection and investigation of cochlear hypoplasia, vestibular dehiscence, partial or total semicircular canal aplasia, dysmorphogenesis and malformations of the vestibulocochlear systems in mutant mouse models that are associated with birth defects related to genetic inner ear disorders in humans, such as in Crouzon syndrome and Pendred syndrome and disorders of the vestibular system [19, 24, 25].

\section{CONFLICT OF INTEREST}

The authors confirm that this article content has no conflict of interest.

\section{ACKNOWLEDGEMENTS}

The authors appreciate the technical assistance of Zsolt Sarnyai, Cyril Monnot and Adam Sierakowiak. We are grateful to Dr. Ann-Christine Eklöf, Department of Comparative Medicine Karolinska Institutet for animal studies support. We thank professor Erik Borg for helpful comments and suggestions. We thank Foundation Tysta Skolan and Afa Insurance for support of this research, and the Harvard Biological Laboratories/MCB for project support.

\section{REFERENCES}

[1] Fitzgerald DC, Mark AS. Endolymphatic duct/sac enhancement on gadolinium magnetic resonance imaging of the inner ear: preliminary observations and case reports. Am J Otol 1996; 17: 603-6.

[2] Casselman JW, Offeciers EF, De Foer B, Govaerts P, Kuhweide R Somers T. CT and MR imaging of congenital abnormalities of the inner ear and internal auditory canal. Eur J Radiol 2001; 40: 94104.

[3] Copeland WR, Hoover JM, Morris JM, Driscoll CL, Link MJ. Use of preoperative MRI to predict vestibular schwannoma intraoperative consistency and facial nerve outcome. J Neurol Surg B Skull Base 2013;74: 347-50.

[4] Naganawa S, Satake H, Kawamura M, Fukatsu H, Sone M, Nakashima T. Separate visualization of endolymphatic space, perilymphatic space and bone by a single pulse sequence; 3Dinversion recovery imaging utilizing real reconstruction after intratympanic Gd-DTPA administration at 3 Tesla. Eur Radiol 2008; 18: 920-4.

[5] Naganawa S, Yamazaki M, Kawai H, Bokura K, Sone M, Nakashima T. Visualization of endolymphatic hydrops in Ménière's disease after intravenous administration of single-dose gadodiamide at 1.5T. Magn Reson Med Sci 2013; 12: 137-9.

[6] Minor LB1, Solomon D, Zinreich JS, Zee DS. Sound- and/or pressure-induced vertigo due to bone dehiscence of the superior semicircular canal. Arch Otolaryngol Head Neck Surg 1998; 124: 249-58.

[7] Lan MY, Shiao JY, Ho CY, Hung HC. Measurements of normal inner ear on computed tomography in children with congenital sensorineural hearing loss. Eur Arch Otorhinolaryngol 2009; 266: 1361-4. 
[8] Czerny C, Gstoettner W, Franz P, Baumgartner WD, Imhof H. CT and MR imaging of acquired abnormalities of the inner ear and cerebellopontine angle. Eur J Radiol 2001; 40: 105-12.

[9] Seemann MD, Beltle J, Heuschmid M, Löwenheim H, Graf H, Claussen CD. Image fusion of CT and MRI for the visualization of the auditory and vestibular system. Eur J Med Res 2005; 10: 47-55.

[10] Counter SA, Bjelke B, Klason T, Chen Z, Borg E. Magnetic resonance imaging of the cochlea, spiral ganglia and eighth nerve of the guinea pig. Neuroreport 1999; 10: 473-9.

[11] Counter SA, Bjelke B, Borg E, Klason T, Chen Z, Duan ML. Magnetic resonance imaging of the membranous labyrinth during in vivo gadolinium (Gd-DTPA-BMA) uptake in the normal and lesioned cochlea. Neuroreport 2000; 11: 3979-83.

[12] Zou J, Zhang W, Poe D, Zhang Y, Ramadan UA, Pyykkö. I. Differential passage of gadolinium through the mouse inner ear barriers evaluated with 4.7T MRI. Hear Res 2010; 259: 36-43.

[13] Counter SA, Nikkhou S, Brené S, et al. MRI evidence of endolymphatic impermeability to the Gadolinium molecule in the in vivo mouse inner ear at 9.4 Tesla. Open Neuroimag J 2013; 28(7): 27-31.

[14] Van Spaendonck MP, Cryns K, Van De Heyning PH, Scheuermann DW, Van Camp G, Timmermans JP. High resolution imaging of the mouse inner ear by microtomography: a new tool in inner ear research. Anat Rec 2000; 259: 229-36.

[15] Lee JH, Park K, Kang TC, Choung YH. Three-dimensional anatomy of the temporal bone in normal mice. Anat Histol Embryol 2009; 38: 311-5.

[16] Heulens I, Suttie M, Postnov A, et al. Craniofacial characteristics of fragile X syndrome in mouse and man. Eur J Hum Genet 2013; 21: 816-23.
[17] Aggarwal M1, Zhang J, Miller MI, Sidman RL, Mori S. Magnetic resonance imaging and micro-computed tomography combined atlas of developing and adult mouse brains for stereotaxic surgery. Neuroscience 2009; 162: 1339-50.

[18] Chan E, Kovacevic N, Ho SK, Henkelman RM, Henderson JT. Development of a high resolution three-dimensional surgical atlas of the murine head for strains $129 \mathrm{~S} 1 / \mathrm{SvImJ}$ and C57BL/6J using magnetic resonance imaging and micro-computed tomography. Neuroscience 2007; 144: 604-15.

[19] Martínez-Abadías N, Motch SM, Pankratz TL, et al.. Tissuespecific responses to aberrant FGF signaling in complex head phenotypes. Dev Dyn 2013; 242: 80-94.

[20] Saito S, Mori Y, Yoshioka Y, Murase K. High-resolution ex vivo imaging in mouse spinal cord using micro-CT with $11.7 \mathrm{~T}-\mathrm{MRI}$ and myelin staining validation. Neurosci Res 2012; 73: 337-40.

[21] Schwarcz A1, Berente Z, Osz E, Dóczi T. In vivo water quantification in mouse brain at 9.4 Tesla in a vasogenic edema model. Magn Reson Med 2000; 46: 1246-9.

[22] Vaughan T1, DelaBarre L, Snyder C, et al. 9.4T human MRI: preliminary results. Magn Reson Med 2006; 56: 1274-82.

[23] Laurell G, Teixeira M, Sterkers O, Ferrary E. Paracellular transport properties of inner ear barriers do not account for cisplatin toxicity in the rat. Hear Res 1997; 110(1-2): 135-40.

[24] Sharghi S, Haghpanah V, Heshmat R, et al. Comparison of MRI findings with traditional criteria in diagnosis of Pendred syndrome. Int J Audiol 2007; 46: 69-74.

[25] Cryns K, van Alphen AM, van Spaendonck MP, et al. Circling behavior in the Ecl mouse is caused by lateral semicircular canal defects. J Comp Neurol 2004; 468: 587-95.

Received: May 09, 2015
(C) Counter et al.; Licensee Bentham Open.

Revised: July 06,2015

Accepted: July 06,2015

This is an open access article licensed under the terms of the Creative Commons Attribution Non-Commercial License (http://creativecommons.org/licenses/by $\mathrm{nc} / 3.0 /$ ), which permits unrestricted, non-commercial use, distribution and reproduction in any medium, provided the work is properly cited. 